\title{
Stage III Kidney Wilms Tumor
}

National Cancer Institute

\section{Source}

National Cancer Institute. Stage III Kidney Wilms Tumor. NCI Thesaurus. Code C7842.

Wilms tumor that is found in areas near the kidney and cannot be completely removed with surgery. The tumor may have spread to nearby organs and blood vessels or throughout the abdomen and to nearby lymph nodes. Lymph nodes are tiny, beanshaped organs that help fight infection. Stage III cancer has not spread outside the abdomen. (National Wilms Tumor Study Group Staging System) 